\title{
A gene for susceptibility to tuberculosis
}

$\mathrm{T}$ he complex genetic nature of what makes some people more susceptible than others to tuberculosis (TB) may finally be revealing itself. The answer may lie in the genes that control cell-mediated immunity, specifically a newly discovered gene that governs how macrophages cope with infectious organisms such as $\mathrm{Myco-}$ bacterium tuberculosis. The discovery sheds new light on the molecular basis of the pathogenesis of $\mathrm{TB}$, a disease that kills more than 2 million people each year.

\section{Why do only some people get tuberculosis?}

It has been known for years that genetics influences our susceptibility to TB. A clear example of this was provided by a terrible accident in 1923, when 249 babies in Lubeck, Germany, were given live $M$. tuberculosis in place of a vaccine. ${ }^{1}$ Of them, 173 survived, which indicated a general resistance to the bacteria. Furthermore, although about one-third of the human population is infected with $M$. tuberculosis, the disease will ever develop in only $10 \%$ of them. ${ }^{1}$ Together, this points to an innate ability of our immune system to defend against $M$. tuberculosis. Macrophages play an important role in the immune response to TB. However, the genes involved in this immunity have been difficult to find.

In 2000, Hui Pan and col- leagues began to tackle this 守 problem. Using a mouse strain known to be very susceptible to TB (death occurs within 4-5 weeks after infection, as compared with 6-8 months for normal mice), the authors identified a region of chromosome 1 that seemed to be responsible for the difference. They termed the region susceptibility to tuberculosis 1 (sst1). However, the specific gene(s) in this region were unknown.

\section{Searching for the genes}

In an attempt to identify the responsible gene(s), Pan and colleagues ${ }^{2}$ replaced the $s s t 1$ region in the TB-susceptible mice with the same region from a normal mouse strain. They found that this "congenic" strain was more resistant to infection with $M$. $t u$ berculosis and better able to control the replication and spread of $M$. tuberculosis.

In addition, the authors isolated a gene in the sst 1 region that was expressed in macrophages from the congenic mice but not in macrophages from the susceptible mice. This gene - the Intracellular pathogen resistance 1 (Ipr1) gene - encodes a protein that appears to be linked to how the macrophages die upon infection. ${ }^{2}$ For example, in susceptible mice, infected macrophages died by necrosis, whereas in congenic mice, they underwent apoptosis (a tightly controlled form of cellular suicide). Therefore, it seems that the Ipr1 gene affects the choice of a cell-death pathway in infected macrophages. Interestingly, this effect also appears to be quite general, since mice expressing the protein could also suppress the growth of Listeria monocytogenes.

The protein that the IprI gene encodes is similar to the human protein SP110b. ${ }^{2}$ Both of these proteins contain domains that suggest they regulate gene expression. In addition, they are both regulated by interferons. Intriguingly, changes in SP110b have been connected to susceptibility to hepatitis C. ${ }^{3}$ This suggests that Ipr1 is involved in how pathogens and host interact, potentially through the regulation of gene expression in macrophages.

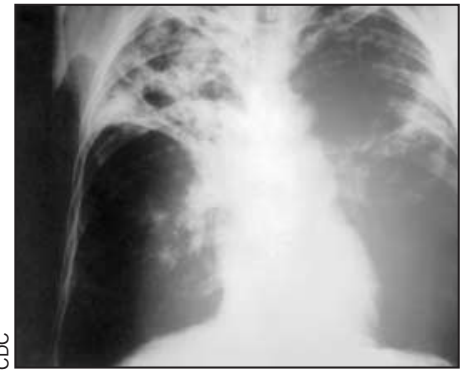

Ipr1 is a novelty in that it appears to provide a mechanism of resistance to 2 different bacteria. This newly discovered mechanism controls the interaction between pathogenesis, cell death and innate immunity. ${ }^{2}$ There are likely factors other than the sst1 region involved in this relationship: although congenic mice were more resistant to $\mathrm{TB}$ than susceptible mice, they were less resistant than normal mice. Regardless, this is an important discovery that awaits confirmation in humans. At least in the case of TB, a disease with immense international importance, understanding this new aspect of innate immunity may lead to new therapeutic targets. David Secko, Vancouver

\section{References}

1. Bellamy R. Susceptibility to mycobacterial infections: the importance of host genetics [review]. Genes Immun 2003;4(1):4-11.

2. Pan H, Yan BS, Rojas M, Shebzukhov YV, Zhou H, Kobzik L, et al. Ipr1 gene mediates innate immunity to tuberculosis [letter]. Nature 2005;434:767-72.

3. Saito T, Ji G, Shinzawa H, Okumoto K, Hattori E, Adachi T, et al. Genetic variations in humans associated with differences in the course of hepatitis C. Biochem Biophys Res Commun 2004;317 (2):335-41. 